poor outcomes in older patients with non-ST-elevation acute coronary syndrome (NSTEACS) undergoing invasive care.

METHOD Older patients undergoing invasive management for NSTEACS were recruited. Cardiovascular status was assessed through evaluation of carotid intima media thickness (CIMT) via B-mode ultrasound (Vivid-I, GE Healthcare), vascular stiffness was assessed through carotid-femoral pulse wave velocity (PWV) (Vicorder device, Skidmore Medical Limited, Bristol, UK), and peripheral arterial endothelial function (EndoPAT 2000 device; Itamar Medical, Caesarea, Israel). Primary outcome consisted of composite of death, myocardial infarction (MI), unplanned revascularisation, stroke and significant bleeding at 1 year.

Results Of the 629 patients screened, 300 patients were recruited into the study. 2 patients had other diagnosis post angiography and were excluded, and a further 1 patient lost to follow-up, meaning sample available for analysis was 297. Overall, 276 (93\%) had non-invasive cardiovascular status assessed at baseline of which 219 (74\%) had CIMT, 276 (93\%) had vascular stiffness and 242 (88\%) had endothelial function data available for analysis. Mean age was $80.5 \pm 4.8$ years and $60.5 \%$ were male. The primary outcome at 1 year was observed in 75 patients $(27.2 \%)$. Cumulative event rates for primary end points were significantly higher in patients with CIMT $0.9 \mathrm{~mm}$ when compared with $<0.9 \mathrm{~mm}(48.4 \%$ vs. 22.9\% Hazard Ratio [HR] 2.39, 95\% Confidence Interval [CI]: 1.33-4.31, P<0.05) driven mainly by bleeding events (Odds Ratio [OR] 5.15, CI: 1.80-14.78, P<0.01). Patients with an abnormal CIMT were 3 times more likely to meet the primary end-point (Odds Ratio OR 3.16, 95\% CI: 1.456.91, $\mathrm{P}<0.01)$, with the association remaining after adjusting for age, gender, frailty and previous MI (OR 3.15, 95\% CI: 1.41-7.07, $\mathrm{P}<0.01$ ). Increased vascular stiffness (PWV $>10 \mathrm{~m} /$

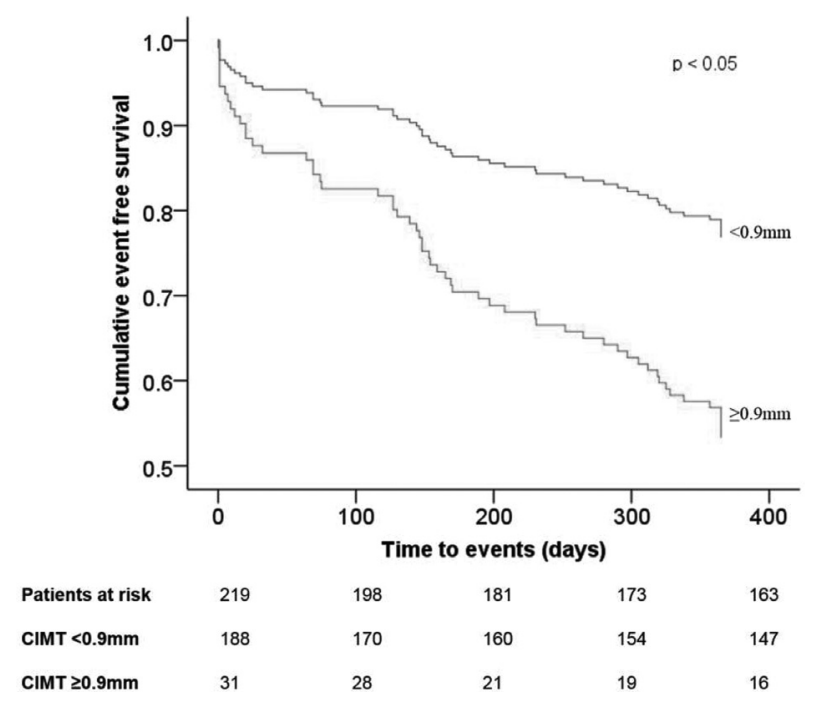

Abstract 161 Figure 1 Cumulative survival rates for the combined end point at 1 year, according to carotid intima-media thickness $\geq 0.9 \mathrm{~mm} v<0.9 \mathrm{~mm}$.

Visualization of cumulative survival rates for the combined end point of death, acute coronary syndrome, unplanned revascularisation, stroke and BARC (Bleeding Academic Research Consortium)-defined bleeding (type 2 or greater) at 1 year, according to carotid intima-media thickness $\geq 0.9 \mathrm{~mm}$ vs. $<0.9 \mathrm{~mm}$. Multivariate analysis using Cox proportional hazards regression model, adjusted for age, gender, frailty and previous myocardial infarction ( $\mathrm{HR} 2.19, \mathrm{Cl}: 1.21-3.98, \mathrm{p}<0.05$ ) s) was associated with a significant reduction in mortality after adjusting for age, gender, frailty and previous MI (OR 0.12, 95\% CI: $0.014-0.98, \quad \mathrm{P}<0.05)$. Endothelial dysfunction as hyperaemia index $<0.40$ vs. 0.40 showed no significant differences in the event rates of combined primary end points adjusting for age, gender, frailty and previous MI $(27.3 \%$ vs. 25.0\%, HR 0.91, 95\% CI: 0.49-1.70, P=0.71).

Conclusion Increased CIMT is associated with adverse outcomes driven by excess bleeding events. Vascular stiffness is associated with reduced mortality in older patients with NSTEACS, even after traditional risk factors for cardiovascular events have been taken into consideration. Endothelial dysfunction is not associated with adverse events. Figure 1.

Conflict of Interest None

\section{RECRUITMENT FOR CLINICAL TRIALS FOR ELDERLY PATIENTS - INSIGHTS FROM THE XIMA AND RINCAL TRIALS}

Rebecca Godfrey*, Adam De Belder. Brighton \& Sussex University Hospitals NHS Trust

\subsection{6/heartjnl-2019-BCS.159}

Introduction The XIMA trial was a randomised comparison of BMS or DES in patients undergoing stenting for CAD in patients aged 80 and over. Recruitment of 800 patients across 20 centres proved straightforward and timely with a high uptake rate in suitable patients.

The RINCAL trial randomised patients to a conservative or an invasive approach for the management of NSTEMI in patients aged 80 and above. Recruitment of 250 patients across 10 sites was laboured and recruitment challenging with participation reluctance.

We determined to understand the causes of this enrolment discrepancy.

Methods Review of the RINCAL study screening log over an 18 -month period at a single high recruiting site, and reasons tabulated for lack of enrolment.

Results During an 18-month period within the RINCAL study at a single site, 102 out of 136 octogenarians with NSTEMI were eligible for inclusion. Of the 34 excluded patients, 14 were due to cognitive impairment [including dementia, lacking capacity to consent to participation, not understanding the patient information sheet], and 20 had concomitant medical comorbidities restricting invasive management.

Of the 102 eligible patients, 24 [24\%] patients participated. 37 [36\%] eligible elderly patients declined to participate. The reasons for declining were: 1) patient declined invasive management, 2) patient wanted invasive management. In 41 [40\%] eligible patients, the managing cardiologist decided that randomisation was not appropriate in one or other of the treatment arms.

Conclusion Evidence is needed to guide management of acute anginal syndromes in older patients. Studies asking simple binary questions have been able to recruit reasonably well [as in the XIMA trial]. Trials asking more complex questions, including RINCAL, have observed high numbers of octogenarians declining to participate. This needs to be borne in mind when designing studies in the elderly, with a greater focus on supporting patients through the consent process.

Conflict of Interest None 